\title{
Weak $\mathcal{Z}$-structures for some classes of groups
}

\author{
CRAig R Guilbault
}

Motivated by the usefulness of boundaries in the study of $\delta$-hyperbolic and CAT( 0$)$ groups, Bestvina introduced a general axiomatic approach to group boundaries, with a goal of extending the theory and application of boundaries to larger classes of groups. The key definition is that of a " $\mathcal{Z}$-structure" on a group $G$. These $\mathcal{Z}$ structures, along with several variations, have been studied and existence results have been obtained for a variety of new classes of groups. Still, relatively little is known about the general question of which groups admit any of the various $\mathcal{Z}$-structures; aside from the (easy) fact that any such $G$ must have type F, ie, $G$ must admit a finite $\mathrm{K}(G, 1)$. In fact, Bestvina has asked whether every type $\mathrm{F}$ group admits a $\mathcal{Z}$-structure or at least a "weak" $\mathcal{Z}$-structure.

In this paper we prove some general existence theorems for weak $\mathcal{Z}$-structures. The main results are as follows.

Theorem A If $G$ is an extension of a nontrivial type $F$ group by a nontrivial type $F$ group, then $G$ admits a weak $\mathcal{Z}$-structure.

Theorem B If $G$ admits a finite $K(G, 1)$ complex $K$ such that the $G$-action on $\tilde{K}$ contains $1 \neq j \in G$ properly homotopic to id $\widetilde{K}$, then $G$ admits a weak $\mathcal{Z}$-structure. Theorem $C$ If $G$ has type $F$ and is simply connected at infinity, then $G$ admits a weak $\mathcal{Z}$-structure.

As a corollary of Theorem $\mathrm{A}$ or $\mathrm{B}$, every type $\mathrm{F}$ group admits a weak $\mathcal{Z}$-structure "after stabilization"; more precisely: if $H$ has type $\mathrm{F}$, then $H \times \mathbb{Z}$ admits a weak $\mathcal{Z}$-structure. As another corollary of Theorem $\mathrm{B}$, every type $\mathrm{F}$ group with a nontrivial center admits a weak $\mathcal{Z}$-structure.

57M07, 20F65; 57N20

\section{Introduction}

Several lines of investigation in geometric topology and geometric group theory seek "nice" compactifications of contractible manifolds or complexes (or ERs/ARs) on which a given group $G$ acts cocompactly as covering transformations. Bestvina [2] has defined a $\mathcal{Z}$-structure and a weak $\mathcal{Z}$-structure on a group $G$ as follows.

A $\mathcal{Z}$-structure on a group $G$ is a pair $(\bar{X}, Z)$ of spaces satisfying: 
(1) $\bar{X}$ is a compact ER.

(2) $Z$ is a $\mathcal{Z}-$ set $^{1}$ in $\bar{X}$.

(3) $X=\bar{X}-Z$ admits a proper, free, cocompact action by $G$.

(4) (Nullity condition) For any open cover $\mathcal{U}$ of $\bar{X}$ and any compactum $K \subseteq X$, all but finitely many $G$-translates of $K$ lie in some element $U$ of $\mathcal{U}$.

If only conditions (1)-(3) are satisfied, $(\bar{X}, Z)$ is called a weak $\mathcal{Z}$-structure on $G$. An additional condition that can be added to conditions (1)-(3), with or without condition (4), is:

(5) The action of $G$ on $X$ extends to an action of $G$ on $\bar{X}$.

Farrell and Lafont [14] refer to a pair $(\bar{X}, Z)$ satisfying (1)-(5) as an EZ-structure. Others have considered pairs that satisfy (1)-(3) and (5); we call those weak $E \mathcal{Z}-$ structures. Depending on the set of conditions satisfied, $Z$ is referred to generically as a boundary for $G$; or more specifically as a $\mathcal{Z}$-boundary, a weak $\mathcal{Z}$-boundary, an EZ-boundary or a weak EZ-boundary.

Example 1 A torsion-free group acting geometrically on a finite-dimensional CAT( 0$)$ space $X$ admits an $E \mathcal{Z}$-structure; one compactifies $X$ by adding the visual boundary. Bestvina and Mess [3] have shown that each torsion-free word hyperbolic group $G$ admits an $E \mathcal{Z}$-structure $(\bar{X}, \partial G)$, where $\partial G$ is the Gromov boundary and $X$ is a Rips complex for $G$. Osajda and Przytycki have shown that systolic groups admit $E \mathcal{Z}-$ structures. Bestvina [2] contains a discussion of $\mathcal{Z}$-structures and weak $\mathcal{Z}$-structures on a variety of other groups, not all of which satisfy condition (5) .

Remark 1 Some authors [11] have extended the above definitions by allowing nonfree $G$-actions (thus allowing for groups with torsion) and by loosening the ER requirement on $\bar{X}$ to that of AR, ie, allowing $\bar{X}$ to be infinite-dimensional. Here we stay with the original definitions, but note that some analogous results are possible in the more general settings.

A group $G$ has type $F$ if it admits a finite $\mathrm{K}(G, 1)$ complex. The following proposition narrows the field of candidates for admitting any sort of $\mathcal{Z}$-structure to those groups of type F.

\footnotetext{
${ }^{1}$ The definition of $\mathcal{Z}$-set, along with definitions of numerous other terms used in the introduction, can be found in Section 2.
} 
Proposition 1.1 If there exists a proper, free, cocompact $G$-action on an $A R Y$, then $G$ has type $F$.

Proof The quotient $q: Y \rightarrow G \backslash Y$ is a covering projection, so $G \backslash Y$ is aspherical and locally homeomorphic to $Y$. By the latter, $G \backslash Y$ is a compact ANR, and thus (by Theorem 2.1) homotopy equivalent to a finite complex. Any such complex is a $\mathrm{K}(G, 1)$.

In [2], Bestvina asked the following pair of questions.

Bestvina's question Does every type $\mathrm{F}$ group admit a $\mathcal{Z}$-structure?

Weak Bestvina's question Does every type $\mathrm{F}$ group admit a weak $\mathcal{Z}$-structure?

The Weak Bestvina question was also posed by Geoghegan in [18, page 425]. Farrell and Lafont [14] have asked whether every type $\mathrm{F}$ group admits an $E \mathcal{Z}$-structure, and the question of which groups admit weak $E \mathcal{Z}$-structures appear in both [3] and [18]. Although interesting special cases abound, a general solution to any of these questions seems out of reach at this time.

As one would expect, the more conditions a $\mathcal{Z}$-structure or its corresponding boundary satisfies, the greater the potential applications. For example, Bestvina has shown that the topological dimension of a $\mathcal{Z}$-boundary is an invariant of the group, it is one less than the cohomological dimension of $G$; this is not true for weak $\mathcal{Z}$-boundaries. But a weak $\mathcal{Z}$-boundary carries significant information about $G$. For example, the Čech cohomology of a weak $\mathcal{Z}$-boundary reveals the group cohomology of $G$ with $\mathbb{Z} G$-coefficients, and the pro-homotopy groups of a weak $\mathcal{Z}$-boundary are directly related to the corresponding end invariants (such as simple connectivity at infinity) of $G$. A weak $\mathcal{Z}$-boundary, when it exists, is well-defined up to shape and can provide a first step toward obtaining a stronger variety of $\mathcal{Z}$-structure on $G . E \mathcal{Z}$ - and weak $E \mathcal{Z}$ boundaries, when they exist, carry the potential for studying $G$ by analyzing its action on the compactum Z. More about these topics can be found in Geoghegan [17; 18], Geohegan and Mihalik [20], Bestvina [2], Farrell and Lafont [14] and the author [23].

In this paper we prove the existence of weak $\mathcal{Z}$-structures for a variety of groups. A notable special case provides a "stabilized solution" to the weak Bestvina question. It asserts that, if $H$ has type $\mathrm{F}$, then $H \times \mathbb{Z}$ admits a weak $\mathcal{Z}$-structure. That result is an easy consequence of either of the following more general theorems, to be proven here.

Theorem 1.2 If $G$ is an extension of a nontrivial type $F$ group by a nontrivial type $F$ group, that is, if there exists a short exact sequence $1 \rightarrow N \rightarrow G \rightarrow Q \rightarrow 1$, where $N$ 
and $Q$ are nontrivial and type $F$, then $G$ admits a weak $\mathcal{Z}$-structure. More generally, if a type $F$ group $G$ is virtually an extension of a nontrivial type $F$ group by a nontrivial type $F$ group, then $G$ admits a weak $\mathcal{Z}$-structure.

Theorem 1.3 Suppose $G$ admits a finite $K(G, 1)$ complex $K$, and the corresponding $G$-action on the universal cover $\tilde{K}$ contains a $1 \neq j \in G$ that is properly homotopic to id $\tilde{\tilde{K}}$. Then $G$ admits a weak $\mathcal{Z}$-structure.

Remark 2 For finite $\mathrm{K}(G, 1)$ complexes $K$ and $L$, or more generally, compact aspherical ANRs with $\pi_{1}(K) \cong G \cong \pi_{1}(L)$, there is a $G$-equivariant proper homotopy equivalence $\tilde{f}: \tilde{K} \rightarrow \widetilde{L}$. If $j \in G$ satisfies the hypothesis of Theorem 1.3 , then so does $\tilde{f} \circ j$. Hence, the existence of such a $j$ can be viewed as a property of $G$, itself.

Example 2 For a closed, orientable, aspherical $n$-manifold $M^{n}$ with $\tilde{M}^{n} \cong \mathbb{R}^{n}$ (eg, $M^{n}$ a Riemannian manifold of nonpositive sectional curvature) every element of $\pi_{1}\left(M^{n}\right)$ satisfies the hypothesis of Theorem 1.2. On the other hand, for finitely generated free groups, no elements do. Of course, weak $\mathcal{Z}$-structures for both of these classes of groups are known for other reasons.

Corollary 1.4 If $H$ is type $F$, then $H \times \mathbb{Z}$ admits a weak $\mathcal{Z}$-structure.

Proof This corollary is immediate from Theorem 1.2. Alternatively, it may be obtained from Theorem 1.3. Let $K$ be a finite $\mathrm{K}(H, 1)$ and $H \times \mathbb{Z}$ act diagonally on $\tilde{K} \times \mathbb{R}$. The nontrivial elements of $\mathbb{Z}$ satisfy the hypotheses of that theorem.

A more general application of Theorem 1.3 is the following.

Corollary 1.5 If $G$ is a type $F$ group with a nontrivial center, then $G$ admits a weak $\mathcal{Z}$-structure.

Proof The point here is that, when $K$ is a finite $\mathrm{K}(G, 1)$, each nontrivial $j \in Z(G)$ satisfies the hypothesis of Theorem 1.3. A complete proof of that fact can be deduced from Gottlieb [22, Theorema II.7]. We sketch an alternative argument.

Let $K^{*}=K \cup A$, where $A$ is an arc with terminal point identified to a vertex $p$ of $K$; let $p^{*}$ be the initial point of $A$. For arbitrary $j \in \pi_{1}(K, p)$ define $f_{j}:\left(K^{*}, p^{*}\right) \rightarrow$ $\left(K^{*}, p^{*}\right)$ to be the identity on $K$; to stretch the initial half of $A$ onto the image copy of $A$; and to send the latter half of $A$ around a loop corresponding to $j$. The induced homomorphism on $\pi_{1}\left(K^{*}, p^{*}\right)$ is conjugation by $j$. If $j \in Z(G)$ that homomorphism is the identity, so $f_{j}$ is homotopic (relative to $p^{*}$ ) to $\mathrm{id}_{K^{*}}$. Since $K^{*}$ is compact, that homotopy lifts to a proper homotopy $\widetilde{F}: \widetilde{K}^{*} \times[0,1] \rightarrow \widetilde{K}^{*}$. Collapse out the preimage of $A \times[0,1]$ in the domain and the preimage of $A$ in the range to get a proper homotopy between id $\tilde{K}$ and the covering translation corresponding to $j$. 
Theorems 1.2 and 1.3 will be obtained from a pair of more general results, with hypotheses more topological than group-theoretic.

Theorem 1.6 Suppose $G$ admits a finite $K(G, 1)$ complex $K$ with the property that $\widetilde{K}$ is proper homotopy equivalent to a product $X \times Y$ of noncompact ANRs, then $G$ admits a weak $\mathcal{Z}$-structure.

Theorem 1.7 Suppose $G$ admits a finite $K(G, 1)$ complex $K$ for which $\tilde{K}$ is proper homotopy equivalent to an ANR $X$ that admits a proper $\mathbb{Z}$-action generated by a homeomorphism $h: X \rightarrow X$ that is properly homotopic to $\operatorname{id}_{X}$. Then $G$ admits a weak $\mathcal{Z}$-structure.

Note that neither the product structure in Theorem 1.6 nor the $\mathbb{Z}$-action in Theorem 1.7 are required to have any relationship to the $G$-action on $\widetilde{K}$.

A third variety of existence theorem for weak $\mathcal{Z}$-structures has, as its primary hypothesis, a condition on the end behavior of $G$.

Theorem 1.8 If $G$ is type $F, 1$-ended and has pro-monomorphic fundamental group at infinity, then $G$ admits a weak $\mathcal{Z}$-structure.

Corollary 1.9 If a type $F$ group $G$ is simply connected at infinity, then $G$ admits a weak $\mathcal{Z}$-structure.

Results found in Jackson [28], Mihalik [30], Profio [31] and Conner and Mihalik [8] show that simple connectivity at infinity is a common property for certain types of group extensions. By applying those results, some interesting overlap can be seen in the collections of groups covered by Corollary 1.9 and those covered by Theorems 1.2 and 1.3 .

In the next section, we introduce some terminology and review a number of established results that are fundamental to later arguments. In Section 3 we prove a variety topological theorems related to end properties of ANRs, complexes and Hilbert cube manifolds. Most importantly, we prove $\mathcal{Z}$-compactifiability for a variety of spaces. Several results obtained there are more general than required for the group-theoretic applications in this paper, and may be of independent interest. In Section 4, we prove the main theorems stated above. In an appendix, we provide an alternative proof, based on the theory of "approximate fibrations," of a crucial lemma from Section 4.

Acknowledgements The author wishes to acknowledge Mike Mihalik and Ross Geoghegan for helpful conversations that led to significant improvements in this paper. Work on this project was aided by a Simons Foundation Collaboration Grant. 


\section{Terminology and background}

\subsection{Inverse sequences of groups}

Throughout this subsection all arrows denote homomorphisms, while arrows of the type $\rightarrow$ or $\ll$ denote surjections and arrows of the type $\succ$ and $\longleftarrow$ denote injections.

Let

$$
G_{0} \stackrel{\lambda_{1}}{\longleftarrow} G_{1} \stackrel{\lambda_{2}}{\longleftarrow} G_{2} \stackrel{\lambda_{3}}{\longleftarrow} \ldots
$$

be an inverse sequence of groups. A subsequence of $\left\{G_{i}, \lambda_{i}\right\}$ is an inverse sequence of the following form:

$$
G_{i_{0}} \stackrel{\lambda_{i_{0}+1} \circ \cdots \circ \lambda_{i_{1}}}{\longleftarrow} G_{i_{1}} \stackrel{\lambda_{i_{1}+1} \circ \cdots \circ \lambda_{i_{2}}}{\longleftarrow} G_{i_{2}} \stackrel{\lambda_{i_{2}+1} \circ \cdots \circ \lambda_{i_{3}}}{\longleftarrow} \cdots
$$

In the future we denote a composition $\lambda_{i} \circ \cdots \circ \lambda_{j}(i \leq j)$ by $\lambda_{i, j}$.

Sequences $\left\{G_{i}, \lambda_{i}\right\}$ and $\left\{H_{i}, \mu_{i}\right\}$ are pro-isomorphic if, after passing to subsequences, there exists a commuting "ladder diagram":

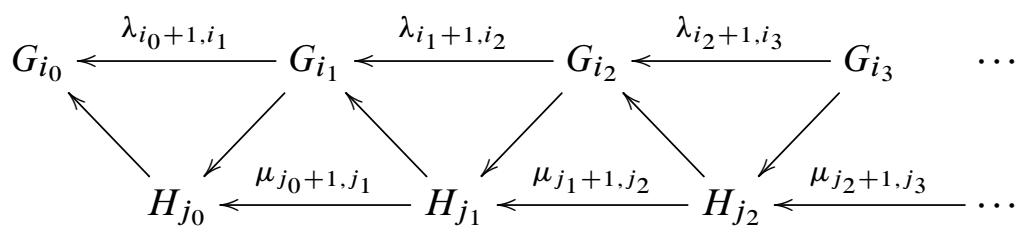

Clearly an inverse sequence is pro-isomorphic to any of its subsequences. To avoid tedious notation, we sometimes do not distinguish $\left\{G_{i}, \lambda_{i}\right\}$ from its subsequences. Instead we assume that $\left\{G_{i}, \lambda_{i}\right\}$ has the desired properties of a preferred subsequence, prefaced by the words "after passing to a subsequence and relabeling."

An inverse sequence $\left\{G_{i}, \lambda_{i}\right\}$ is called pro-monomorphic if it is pro-isomorphic to an inverse sequence of monomorphisms and pro-epimorphic (more commonly called semistable or Mittag-Leffler) if it is pro-isomorphic to an inverse sequence of epimorphisms. It is stable if it is pro-isomorphic to a constant inverse sequence $\left\{H, \mathrm{id}_{H}\right\}$, or equivalently, to an inverse sequence of isomorphisms. It is a standard fact that $\left\{G_{i}, \lambda_{i}\right\}$ is stable if and only if it is both pro-monomorphic and pro-epimorphic.

A few more special classes of inverse sequences will be of interest in this paper. A sequence that is pro-isomorphic to the trivial sequence $1 \leftarrow 1 \leftarrow 1 \leftarrow \cdots$ is called pro-trivial; a sequence pro-isomorphic to an inverse sequence of finitely generated groups is called pro-finitely generated; and a sequence that is pro-isomorphic to an inverse sequence of free groups is called pro-free. A sequence that is both pro-finitely 
generated and pro-free is easily seen to be pro-isomorphic to an inverse sequence of finitely generated free groups. We call such a sequence pro-finitely generated free.

The inverse limit of a sequence $\left\{G_{i}, \lambda_{i}\right\}$ is the subgroup of $\prod G_{i}$ defined by

$$
\lim _{\longleftarrow}\left\{G_{i}, \lambda_{i}\right\}=\left\{\left(g_{0}, g_{1}, g_{2}, \ldots\right) \in \prod_{i=0}^{\infty} G_{i} \mid \lambda_{i}\left(g_{i}\right)=g_{i-1}\right\} .
$$

In the special case where $\left\{G_{i}, \lambda_{i}\right\}$ is an inverse sequence of abelian groups, we also define the derived limit $^{2}$ to be the following quotient group:

$$
\lim ^{1}\left\{G_{i}, \lambda_{i}\right\}=\left(\prod_{i=0}^{\infty} G_{i}\right) /\left\{\left(g_{0}-\lambda_{1} g_{1}, g_{1}-\lambda_{2} g_{2}, g_{2}-\lambda_{3} g_{3}, \ldots\right) \mid g_{i} \in G_{i}\right\}
$$

It is a standard fact that pro-isomorphic inverse sequences of groups have isomorphic inverse limits and, pro-isomorphic inverse sequences of abelian groups have isomorphic derived limits.

\subsection{Absolute neighborhood retracts}

Throughout this paper, all spaces are assumed to be separable metric. A locally compact space $X$ is an ANR (absolute neighborhood retract) if it can be embedded into $\mathbb{R}^{n}$ or, if necessary, $\mathbb{R}^{\infty}$ (a countable product of real lines) as a closed set in such a way that there exists a retraction $r: U \rightarrow X$, where $U$ is a neighborhood of $X$. If the entire space $\mathbb{R}^{n}$ or $\mathbb{R}^{\infty}$ retracts onto $X$, we call $X$ an AR (absolute retract). If $X$ is finite-dimensional, all mention of $\mathbb{R}^{\infty}$ can be omitted. A finite-dimensional ANR is called an ENR (Euclidean neighborhood retract) and a finite-dimensional AR an ER. With a little effort it can be shown that an AR (respectively, ER) is simply a contractible ANR (respectively, ENR).

A space $X$ is locally contractible if every neighborhood $U$ of a point $x \in X$ contains a neighborhood $V$ of $x$ that contracts within $U$. It is a standard fact that every ANR is locally contractible. For finite-dimensional spaces, that property characterizes ANRs. In other words, a locally compact, finite-dimensional space $X$ is an ANR (and hence an ENR) if and only if it is locally contractible. It follows that every finite-dimensional locally finite polyhedron or CW complex is an ENR; if it is contractible, it is an ER.

The following famous result will be used in this paper.

Theorem 2.1 (West [35]) Every ANR is homotopy equivalent to a locally finite polyhedron. Every compact ANR is homotopy equivalent to a finite polyhedron.

\footnotetext{
${ }^{2}$ The definition of derived limit can be generalized to include nonableian groups [18, Section 11.3], but that will not be needed in this paper.
} 


\subsection{Proper maps and homotopies}

When working with noncompact space, the notion of "properness" is crucial. A map $f: X \rightarrow Y$ is proper if $f^{-1}(C)$ is compact whenever $C \subseteq Y$ is compact. Maps $f_{0}, f_{1}: X \rightarrow Y$ are properly homotopic, denoted by $f_{0} \stackrel{p}{\simeq} f_{1}$, if there exists a proper map $H: X \times[0,1] \rightarrow Y$ with $H_{0}=f_{0}$ and $H_{1}=f_{1}$. Spaces $X$ and $Y$ are proper homotopy equivalent, denoted by $\underset{p}{\underset{p}{\simeq}} \underset{\sim}{\simeq}$, if there exist proper maps $f: X \rightarrow Y$ and $g: Y \rightarrow X$ with $g f \stackrel{p}{\simeq} \mathrm{id}_{X}$ and $f g \stackrel{p}{\simeq} \operatorname{id}_{Y}$.

\subsection{Ends of spaces and the fundamental group at infinity}

A subset $N$ of a space $X$ is a neighborhood of infinity if $\overline{X-N}$ is compact. A standard argument shows that, when $X$ is an ANR and $C$ is a compact subset of $X, X-C$ has at most finitely many unbounded components, ie, finitely many components with noncompact closures. If $X-C$ has both bounded and unbounded components, the situation can be simplified by letting $C^{\prime}$ consist of $C$ together with all bounded components. Then $C^{\prime}$ is compact, and $X-C^{\prime}$ consists entirely of unbounded components.

We say that $X$ has $k$ ends if there exists a compactum $C \subseteq X$ such that, for every compactum $D$ with $C \subset D, X-D$ has exactly $k$ unbounded components. When $k$ exists, it is uniquely determined; if $k$ does not exist, we say $X$ has infinitely many ends. Thus, a space is 0 -ended if and only if $X$ is compact, and 1-ended if and only if it contains arbitrarily small connected neighborhoods of infinity.

A nested sequence $N_{0} \supseteq N_{1} \supseteq N_{2} \supseteq \cdots$ of neighborhoods of infinity, with each $N_{i} \subseteq$ int $N_{i-1}$, is cofinal if $\bigcap_{i=0}^{\infty} N_{i}=\varnothing$. Such a sequence is easily obtained: choose an exhaustion of $X$ by compacta $C_{0} \subseteq C_{1} \subseteq C_{2} \subseteq \cdots$, with $C_{i-1} \subseteq$ int $C_{i}$; then let $N_{i}=X-C_{i}$. When closed neighborhoods of infinity are required, let $N_{i}=\overline{X-C_{i}}$.

Given a nested cofinal sequence $\left\{N_{i}\right\}_{i=0}^{\infty}$ of neighborhoods of infinity, base points $p_{i} \in N_{i}$ and paths $r_{i} \subset N_{i}$ connecting $p_{i}$ to $p_{i+1}$, we obtain an inverse sequence:

$$
\pi_{1}\left(N_{0}, p_{0}\right) \stackrel{\lambda_{1}}{\longleftarrow} \pi_{1}\left(N_{1}, p_{1}\right) \stackrel{\lambda_{2}}{\longleftarrow} \pi_{1}\left(N_{2}, p_{2}\right) \stackrel{\lambda_{3}}{\longleftarrow} \cdots
$$

Here, each $\lambda_{i+1}: \pi_{1}\left(N_{i+1}, p_{i+1}\right) \rightarrow \pi_{1}\left(N_{i}, p_{i}\right)$ is the homomorphism induced by inclusion followed by the change of base point isomorphism determined by $r_{i}$. The proper ray $r:[0, \infty) \rightarrow X$ obtained by piecing together the $r_{i}$ in the obvious manner is referred to as the base ray for the inverse sequence, and the pro-isomorphism class of the inverse sequence is called the fundamental group at infinity of $X$ based at $r$ and is denoted pro- $\pi_{1}(\varepsilon(X), r)$. It is a standard fact that pro- $\pi_{1}(X, r)$ is independent of the sequence of neighborhoods $\left\{N_{i}\right\}$ or the base points, provided those base points tend to infinity along the ray $r$, and corresponding subpaths of $r$ are used in defining the $\lambda_{i}$. 
More generally, pro- $\pi_{1}(\varepsilon(X), r)$ depends only upon the proper homotopy class of $r$. If $X$ is 1 -ended and pro- $\pi_{1}(\varepsilon(X), r)$ is semistable for some proper ray $r$, it can be shown that all proper rays in $X$ are properly homotopic; in that case we say that $X$ is strongly connected at infinity. When $X$ is strongly connected at infinity, it is safe to omit mention of the base ray and to speak generally of the fundamental group at infinity of $X$, and denote it by pro- $\pi_{1}(\varepsilon(X))$. If $X$ is 1 -ended and pro- $\pi_{1}(\varepsilon(X), r)$ is pro-trivial, we call $X$ simply connected at infinity.

The fundamental group at infinity is clearly not a homotopy invariant of a space, but it is a proper homotopy invariant. More precisely, if $f: X \rightarrow Y$ is a proper homotopy equivalence, then pro- $\pi_{1}(\varepsilon(X), r)$ is pro-isomorphic to pro- $\pi_{1}(\varepsilon(Y), f \circ r)$.

For a group $G$ of type $\mathrm{F}$, the universal cover $\tilde{K}$ of a finite $\mathrm{K}(G, 1)$ complex $K$ is well-defined up to proper homotopy type. So the number of ends of $G$ is welldefined; and if $\widetilde{K}$ is 1 -ended, except for the issue of a base ray, we may view pro$\pi_{1}(\varepsilon(\widetilde{K}), r)$ as an invariant of $G$. The base ray issue goes away when pro- $\pi_{1}(\varepsilon(\widetilde{K}), r)$ is semistable, so there is no ambiguity in defining a 1 -ended $G$ to have semistable, stable or trivial fundamental group at infinity, according to whether pro- $\pi_{1}(\varepsilon(\widetilde{K}), r)$ has the corresponding property. With some additional work, it can be shown that the property of pro- $\pi_{1}(\varepsilon(\widetilde{K}), r)$ being pro-monomorphic is also independent of base ray and, thus, attributable to $G$. See Geohegan and the author [19, Section 2] for further discussion.

Although not needed for this paper, the requirement in the previous paragraph, that $G$ have type F can be significantly weakened. In particular, if $G$ is finitely presented, and $L$ is any finite complex with fundamental group $G$, then the number of ends of $\widetilde{L}$ and the properties of pro- $\pi_{1}(\varepsilon(\tilde{L}), r)$ discussed above, are invariants of $G$. Thus, for example, a finitely presented group $G$ is called simply connected at infinity if $\widetilde{L}$ has that property. For more information about the fundamental group at infinity of spaces and groups, including proofs of the asserstions made in this section; see [18] or [23].

\subsection{Finite domination and inward tameness}

A space $Y$ has finite homotopy type if it is homotopy equivalent to a finite CW complex; it is finitely dominated if there is a finite complex $K$ and maps $u: Y \rightarrow K$ and $d: K \rightarrow Y$ such that $d \circ u \simeq \operatorname{id}_{Y}$. If $Y$ is an ANR, then $Y$ is finitely dominated if and only if there exists a self-homotopy that "pulls $Y$ into a compact subset," ie, $H: Y \times[0,1] \rightarrow Y$ such that $H_{0}=\mathrm{id}_{Y}$ and $\overline{H_{1}(Y)}$ is compact. This equivalence is easily verified when (for example) $K$ is a locally finite polyhedron; a discussion of the general case can be found in [23, Section 3.4].

The following clever observation will be used later. 
Theorem 2.2 (Mather [29]) If a space $Y$ is finitely dominated, then $Y \times \mathbb{S}^{1}$ has finite homotopy type.

An ANR $X$ is inward tame if, for every closed neighborhood of infinity $N$ in $X$, there is a homotopy $K: N \times[0,1] \rightarrow N$ with $K_{0}=\mathrm{id}_{N}$ and $\overline{K_{1}(N)}$ compact (a homotopy pulling $N$ into a compact subset). By an easy application of Borsuk's homotopy extension property, this is equivalent to the existence of a cofinal sequence $\left\{N_{i}\right\}$ of closed neighborhoods of infinity, each of which can be pulled into a compact set. If $X$ contains a cofinal sequence $\left\{N_{i}\right\}$ of closed ANR neighborhoods ${ }^{3}$ of infinity, then inward tameness is equivalent to each of those (hence, all closed ANR neighborhoods of infinity) being finitely dominated. The author [23, Section 3.5] provides additional details.

Inward tameness is an invariant of proper homotopy type. Roughly speaking, if $f: X \rightarrow Y$ and $g: Y \rightarrow X$ are proper homotopy inverses and $H$ is a homotopy that pulls a neighborhood of infinity of $X$ into a compact set, then $f \circ H_{t} \circ g$ pulls a neighborhood of $Y$ into a compact set. More details can be found in [23, Section 3.5].

\subsection{Some basic $K$-theory}

An important result from Wall [34] asserts that, for each finitely dominated, connected space $Y$, there is a well-defined obstruction $\sigma(Y)$, lying in the reduced projective class group $\widetilde{K}_{0}\left(\mathbb{Z}\left[\pi_{1}(Y)\right]\right)$, which vanishes if and only if $Y$ has finite homotopy type.

A related algebraic construction is the Whitehead group. If $(A, B)$ is a pair of connected, finite $\mathrm{CW}$ complexes and $B \hookrightarrow A$ is a homotopy equivalence, then there is a welldefined obstruction $\tau(B)$, lying in an abelian group $\mathrm{Wh}\left(\pi_{1}(B)\right)$ that vanishes if and only if $B \hookrightarrow A$ is a simple homotopy equivalence. Definitions and details can be found in Cohen [7].

Both of the above algebraic constructs act as functors in the sense that, if $\lambda: G \rightarrow H$ is a group homomorphism, there are naturally induced homomorphisms $\lambda_{*}: \widetilde{K}_{0}(\mathbb{Z}[G]) \rightarrow$ $\widetilde{K}_{0}(\mathbb{Z}[G])$ and $\lambda_{*}: \mathrm{Wh}(G) \rightarrow \mathrm{Wh}(H)$.

For the purposes of this paper, the main thing we need to know about $\tilde{K}_{0}\left(\mathbb{Z}\left[\pi_{1}(Y)\right]\right)$ or $\mathrm{Wh}\left(\pi_{1}(B)\right)$ is contained in a famous result of Bass, Heller and Swan [1].

Theorem 2.3 If $G$ is a finitely generated free group, then both $\tilde{K}_{0}(\mathbb{Z}[G])$ and $\mathrm{Wh}(G)$ are the trivial group.

\footnotetext{
${ }^{3}$ In this case, $X$ is called sharp at infinity. Most commonly arising ANRs (for example: locally finite polyhedra, manifolds, proper CAT(0) spaces and Hilbert cube manifolds) are sharp at infinity.
} 


\subsection{Mapping cylinders, mapping tori and mapping telescopes}

For any map $f: K \rightarrow L$ and closed interval $[a, b]$, the mapping cylinder $\mathcal{M}_{[a, b]}(f)$ is the quotient space $L \sqcup(K \times[a, b]) / \sim$, where $\sim$ is the equivalence relation generated by the rule $(x, a) \sim f(x)$ for all $x \in K$. Let $q_{[a, b]}: L \sqcup(K \times[a, b]) \rightarrow \mathcal{M}_{[a, b]}(f)$ be the quotient map. Then, for each $r \in(a, b], q_{[a, b]}$ restricts to an embedding of $K \times\{r\}$ into $\mathcal{M}_{[a, b]}(f)$; denote the image of $K \times\{r\}$ by $K_{r}$. The quotient map is also an embedding when restricted to $L$; let $L_{a} \subseteq \mathcal{M}_{[a, b]}(f)$ be that copy of $L$. We call $K_{b}$ the domain end and $L_{a}$ the range end of $\mathcal{M}_{[a, b]}(f)$. Note the existence of a projection map $p_{[a, b]}: \mathcal{M}_{[a, b]}(f) \rightarrow[a, b]$ for which $p_{[a, b]}^{-1}(r)=K_{r}$ is a copy of $K$ for each $r \in(a, b]$ and $p_{[a, b]}^{-1}(a)=L_{a}$ is a copy of $L$. Note also that, when $K=L$, ie, $f$ maps $K$ to itself, all of the above still applies. In that case, each point preimage of $p_{[a, b]}$ is a copy of $K$, but the copy $K_{a}$ differs from the others, in that it is not necessarily parallel to neighboring copies.

Remark 3 Clearly the topological type of $\mathcal{M}_{[a, b]}(f)$ does not depend on the interval $[a, b]$, and for most purposes can be taken to be $[0,1]$. But in the treatment that follows, it will be useful to allow the interval to vary.

The following standard application of mapping cylinders will be used several times in this paper. A proof, in which properness is not mentioned, can be found in Dugundji [12, page 372]. For our purposes, it is only the easy (converse) direction of the proper assertion that will be used.

Lemma 2.4 A map $f: K \rightarrow L$ between ANRs is a homotopy equivalence if and only if there exists a strong deformation retraction of $\mathcal{M}_{[a, b]}(f)$ onto $M_{b}$. It is a proper homotopy equivalence if and only if there exists a proper strong deformation retraction of $\mathcal{M}_{[a, b]}(f)$ onto $K_{b}$.

The bi-infinite mapping telescope of a map $f: K \rightarrow K$ is obtained by gluing together infinitely many mapping cylinders. More precisely,

$\operatorname{Tel}_{f}(K)=\cdots \cup \mathcal{M}_{[-2,-1]}(f) \cup \mathcal{M}_{[-1,0]}(f) \cup \mathcal{M}_{[0,1]}(f) \cup \mathcal{M}_{[1,2]}(f) \cup \mathcal{M}_{[2,3]}(f) \cup \cdots$

where the gluing is accomplished by identifying the domain end of each $\mathcal{M}_{[n-1, n]}(f)$ with the range end of $\mathcal{M}_{[n, n+1]}(f)$. Notationally, this works well since, under the convention described above, each is denoted $K_{n}$. Projection maps may be pieced together to obtain a projection $p$ : $\operatorname{Tel}_{f}(K) \rightarrow \mathbb{R}$, for which $p^{-1}(r)=K_{r}$ is a copy of $K$, for each $r \in \mathbb{R}$. A schematic of $\operatorname{Tel}_{f}(K)$ is contained in Figure 1 of Appendix A.1. 
The mapping torus of $f: K \rightarrow K$ is obtained from $\mathcal{M}_{[0,1]}(f)$ by identifying $K_{0}$ with $K_{1}$. It may also be defined more directly as the quotient space

$$
\operatorname{Tor}_{f}(K)=K \times[0,1] / \sim,
$$

where $\sim$ is the equivalence relation generated by $(x, 0) \sim(f(x), 1)$ for each $x \in K$. The following facts about mapping tori are standard.

Lemma 2.5 Let $K$ be a connected ANR, $f:(K, p) \rightarrow(K, q)$ a map that induces an isomorphism $\varphi: \pi_{1}(K, p) \rightarrow \pi_{1}(K, q)$ and $\lambda$ a path in $K$ from $q$ to $p$. Then:

(1) $\pi_{1}\left(\operatorname{Tor}_{f}(K),(p, 0)\right) \cong \pi_{1}(K, p) \rtimes_{\varphi}\langle t\rangle$, where $t$ is an infinite order element represented by the loop $(\{p\} \times[0,1]) \cdot \lambda$.

(2) The infinite cyclic cover of $\operatorname{Tor}_{f}(K)$ corresponding to the projection mapping $\pi_{1}(K, p) \rtimes_{\varphi}\langle t\rangle \rightarrow\langle t\rangle$ is the bi-infinite mapping telescope $\operatorname{Tel}_{f}(K)$.

The following fact about mapping tori can be found in [19], where it plays a crucial role. We will make significant use of it here as well.

Lemma 2.6 Let $X$ be a connected ANR that admits a proper $\mathbb{Z}$-action generated by a homeomorphism $j: X \rightarrow X$. Then $(\langle j\rangle \backslash X) \times \mathbb{R}$ is homeomorphic to $\operatorname{Tor}_{j}(X)$.

\subsection{Hilbert cube manifolds}

The Hilbert cube is the infinite product

$$
\mathcal{Q}=\prod_{i=1}^{\infty}[-1,1] \quad \text { with metric } d\left(\left(x_{i}\right),\left(y_{i}\right)\right)=\sum_{i=1}^{\infty} \frac{\left|x_{i}-y_{i}\right|}{2^{i}} .
$$

A Hilbert cube manifold is a space $X$ with the property that each $x \in X$ has a neighborhood homeomorphic to $\mathcal{Q}$. Although we are primarily interested in finitedimensional spaces, Hilbert cube manifolds play a key role in this paper. A pair of classical results will allow us to move between the categories of ANRs and locally finite polyhedra through the use of Hilbert cube manifolds.

Theorem 2.7 (Edwards [13]) If $A$ is an $A N R$, then $A \times \mathcal{Q}$ is a Hilbert cube manifold.

Theorem 2.8 (Chapman [5]) If $X$ is a Hilbert cube manifold, then there is a locally finite polyhedron $K$ for which $X \approx K \times \mathcal{Q}$. 


\section{$2.9 \mathcal{Z}$-sets and $\mathcal{Z}$-compactifications}

A closed subset $A$ of an ANR $Y$ is a $\mathcal{Z}$-set if either of the following equivalent conditions is satisfied:

- There exists a homotopy $H: Y \times[0,1] \rightarrow Y$ such that $H_{0}=\operatorname{id}_{Y}$ and $H_{t}(X) \subseteq$ $Y-A$ for all $t>0$. (We say that $H$ instantly homotopes $Y$ off from $A$.)

- For every open set $U$ in $Y, U-A \hookrightarrow U$ is a homotopy equivalence.

A $\mathcal{Z}$-compactification of a space $X$ is a compactification $\bar{X}=X \sqcup Z$ with the property that $Z$ is a $\mathcal{Z}$-set in $\bar{X}$. In this case, $Z$ is called a $\mathcal{Z}$-boundary for $X$. Implicit in this definition is the requirement that $\bar{X}$ be an ANR; moreover, since an open subset of an ANR is an ANR, $X$ itself must be an ANR to be a candidate for $\mathcal{Z}$-compactification. Hanner's Theorem [27] ensures that every compactification $\bar{X}$ of an ANR $X$, for which $\bar{X}-X$ satisfies either of the "negligibility conditions" in the definition of $\mathcal{Z}$-set, is necessarily an ANR; hence, it is a $\mathcal{Z}$-compactification. By a similar (but much easier) result in dimension theory, $\mathcal{Z}$-compactification does not increase dimension; so, if $X$ is an ENR, so is $\bar{X}$.

Example 3 The compactification of $\mathbb{R}^{n}$ obtained by adding the $(n-1)$-sphere at infinity is the prototypical $\mathcal{Z}$-compactification. More generally, addition of the visual boundary to a proper $\mathrm{CAT}(0)$ space is a $\mathcal{Z}$-compactification. In [3], it is shown that, for a torsion-free $\delta$-hyperbolic group $G$, an appropriately chosen Rips complex can be $\mathcal{Z}$-compactified by adding the Gromov boundary $\partial G$.

The purely topological question of when an ANR, an ENR, or even a locally finite polyhedron admits a $\mathcal{Z}$-compactification is an open question (see the author [24]). However, Chapman and Siebenmann [6] have given a complete classification of $\mathcal{Z}$-compactifiability for Hilbert cube manifolds. That result, in combination with Theorem 2.7, has substantial implications for the general case.

Here we provide a slightly simplified version of the Chapman-Siebenmann Theorem. We state the result only for 1 -ended Hilbert cube manifolds $X$, since that is all we need in this paper. We also simplify the definitions of $\sigma_{\infty}(X)$ and $\tau_{\infty}(X)$ by basing them on a prechosen nested, cofinal sequence of nice neighborhoods of infinity. It is true, but would take some time, to explain why the resulting obstructions do not depend on that choice.

A particularly nice variety of closed neighborhood of infinity $N \subseteq X$ is one that is, itself, a Hilbert cube manifold and whose topological boundary $\operatorname{Bd}_{X} N$ is a Hilbert 
cube manifold with a neighborhood in $X$ homeomorphic to $\operatorname{Bd}_{X} N \times[-1,1]$. Call such neighborhoods of infinity clean. By applying Theorems 2.7 and 2.8, clean neighborhoods of infinity are easily found.

Theorem 2.9 (Chapman-Siebenmann) Let $X$ be a 1-ended Hilbert cube manifold and $\left\{N_{i}\right\}$ a nested cofinal sequence of connected clean neighborhoods of infinity. Then $X$ admits a $\mathcal{Z}$-compactification if and only if each of the following conditions holds:

(a) $X$ is inward tame.

(b) $\sigma_{\infty}(X) \in \lim _{\longleftarrow}\left\{\tilde{K}_{0}\left(\mathbb{Z} \pi_{1}\left(N_{i}\right)\right), \lambda_{i *}\right\}$ is zero.

(c) $\tau_{\infty}(X) \in \lim ^{1}\left\{\mathrm{Wh}\left(\pi_{1}\left(N_{i}\right)\right), \lambda_{i *}\right\}$ is zero.

The inverse sequences $\left\{\widetilde{K}_{0}\left(\mathbb{Z} \pi_{1}\left(N_{i}\right)\right), \lambda_{i *}\right\}$ and $\left\{\mathrm{Wh}\left(\pi_{1}\left(N_{i}\right)\right), \lambda_{i *}\right\}$ in conditions (b) and (c) are obtained by applying the $\widetilde{K}_{0}$-functor and the Wh-functor to sequence (2-2). The obstruction $\sigma_{\infty}(X)$ is just the sequence $\left(\sigma\left(N_{0}\right), \sigma\left(N_{1}\right), \sigma\left(N_{2}\right), \ldots\right)$ of Wall finiteness obstructions of the $N_{i}$. Condition (a) ensures that each $N_{i}$ is finitely dominated, so the individual obstructions are all defined; without condition (a), there is no condition (b). Similarly, condition (c) requires condition (b). It is related to the Whitehead torsion of inclusions $\mathrm{Bd}_{X} N_{i} \hookrightarrow \overline{N_{i}-N_{i+1}}$, after the $N_{i}$ have been improved significantly so that those inclusions are homotopy equivalences. The reader should consult [6] for details or [23, Section 8.2] for a less formal discussion of Theorem 2.9. For our purposes, those details are not so important since the obstructions arising here will be shown to vanish by straightforward applications of Theorem 2.3.

Remark 4 Condition (a) makes sense for an arbitrary ANR $X$. If $X$ satisfies (a) and is sharp at infinity, then condition (b) also makes immediate sense; it is satisfied if and only if $X$ contains arbitrarily small closed ANR neighborhoods of infinity having finite homotopy type. Condition (c) is more problematic; even when (a) and (b) are satisfied, if $X$ is not a Hilbert cube manifold, it may be impossible to find neighborhoods of infinity $N_{i}$ with each $\mathrm{Bd}_{X} N_{i} \hookrightarrow \overline{N_{i}-N_{i+1}}$ a homotopy equivalence; an example can be found in the author and Tinsley [26]. The solution to this problem is to define the obstructions for an ANR $X$ to be the corresponding obstructions for the Hilbert cube manifold $X \times \mathcal{Q}$. Then (a)-(c) are necessary for $\mathcal{Z}$-compactifiability of $X$; unfortunately, they are not sufficient. The author [24] exhibits a locally finite 2-dimensional polyhedron $K$ that satisfies (a)-(c), but is not $\mathcal{Z}$-compactifiable. A suitable characterization of $\mathcal{Z}$-compactifiable ANRs is an open question. 
For an ANR $X$, Theorem 2.9 allows us to determine whether $X \times \mathcal{Q}$ is $\mathcal{Z}$-compactifiable. The following result, with $\mathbb{I}=[-1,1]$, frequently allows us to restore finite-dimensionality.

Theorem 2.10 (Ferry [15]) If $K$ is a finite-dimensional locally finite polyhedron and $K \times \mathcal{Q}$ is $\mathcal{Z}$-compactifiable, then $K \times \mathbb{I}^{2 \cdot \operatorname{dim} K+5}$ is $\mathcal{Z}$-compactifiable.

\section{Topological results}

In this section we prove a variety of topological results that are primary ingredients in the proofs of our main theorems. We have broken the section into three parts: the first contains results about product spaces; the second deals with spaces that admit a proper $\mathbb{Z}$-action generated by a homeomorphism properly homotopic to the identity; and the third looks at spaces that are simply connected at infinity.

\subsection{Products of noncompact spaces}

Lemma 3.1 Let $X$ be an ANR that is finitely dominated. Then $X \times \mathbb{R}$ is inward tame.

Proof Since inward tameness is an invariant of proper homotopy type, we may use Theorem 2.1 to reduce to the case that $X$ is a locally finite polyhedron. For that case, the proof given by the author [25, Proposition 3.1] for open manifolds is valid, with only minor modifications. With a few additional modifications, the appeal to Theorem 2.1 can be eliminated.

The next lemma requires a new definition. We say that an ANR $X$ is movably finitely dominated if, for every neighborhood of infinity $N \subseteq X$, there is a self-homotopy of $X$ that pulls $X$ into a compact subset of $N$, ie, $H: X \times[0,1] \rightarrow X$ such that $H_{0}=\mathrm{id}_{X}$ and $\overline{H_{1}(X)}$ is compact and contained in $N$. The motivation for this definition becomes immediately clear in the following lemma. The most important examples are the simplest; every contractible ANR is movably finitely dominated, since it is dominated by each singleton subset.

Lemma 3.2 Let $X$ and $Y$ be connected, noncompact, movably finitely dominated ANRs. Then $X \times Y$ is inward tame.

Proof Let $A \subseteq X$ and $B \subseteq Y$ be compact and $N=\overline{(X \times Y)-(A \times B)}$ the corresponding closed neighborhood of infinity. It suffices to prove the following claim. 
Claim There exits a homotopy $J: N \times[0,1] \rightarrow N$ with $J_{0}=\mathrm{id}_{N}$ and $\overline{J_{1}(N)}$ compact.

Choose compact $A^{\prime} \subseteq X$ and $B^{\prime} \subseteq Y$ such that $A \subseteq$ int $_{X} A^{\prime}$ and $B \subseteq$ int $_{X} B^{\prime}$, and let $\lambda: X \rightarrow[0,1]$ and $\mu: Y \rightarrow[0,1]$ be Urysohn functions with $\lambda(A)=0=\mu(B)$ and $\lambda\left(\overline{X-A^{\prime}}\right)=1=\mu\left(\overline{Y-B^{\prime}}\right)$. Then choose compact $K \subseteq X-A^{\prime}$ and $L \subseteq Y-B^{\prime}$ along with homotopies $F: X \times[0,1] \rightarrow X$ such that $F_{0}=\operatorname{id}_{X}$ and $F_{1}(X) \subseteq K$ and $G: Y \times[0,1] \rightarrow Y$ such that $G_{0}=\operatorname{id}_{Y}$ and $G_{1}(X) \subseteq L$.

We will build a homotopy $H$ that pulls $X \times Y$ into a compact subset while fixing $A \times B$. By arranging that tracks of points from $N$ stay in $N$, the restriction of this homotopy will satisfy the claim.

Define $\hat{F}: X \times Y \times[0,1] \rightarrow X \times Y$ by $\widehat{F}(x, y, t)=(F(x, \mu(y) \cdot t), y)$ and note that:

- $\widehat{F}_{1}(X \times Y) \subseteq\left(X \times B^{\prime}\right) \cup(K \times Y)$

- $\left.\widehat{F}_{t}\right|_{X \times B}=$ id for all $t$

- tracks of points in $N$ stay in $N$

Similarly, let $\widehat{G}: X \times Y \times[0,1] \rightarrow X \times Y$ by $\widehat{G}(x, y, t)=(x, G(y, \lambda(x) \cdot t))$ and note that:

- $\widehat{G}_{1}(X \times Y) \subseteq\left(A^{\prime} \times Y\right) \cup(X \times L)$

- $\left.\widehat{G}\right|_{A \times Y}=\mathrm{id}$

- tracks of points in $N$ stay in $N$

Now define $H: X \times Y \times[0,1] \rightarrow X \times Y$ by the rule

$$
H_{t}= \begin{cases}\widehat{F}_{3 t} & 0 \leq t \leq \frac{1}{3}, \\ \widehat{G}_{3 t-1} \circ \widehat{F}_{1} & \frac{1}{3} \leq t \leq \frac{2}{3}, \\ \widehat{F}_{3 t-2} \circ \widehat{G}_{1} \circ \widehat{F}_{1} & \frac{2}{3} \leq t \leq 1 .\end{cases}
$$

A quick check shows that $H_{1}(X \times Y)$ is contained in the compact set $\widehat{F}_{1} \widehat{G}_{1}\left(A^{\prime} \times B^{\prime}\right) \cup$ $(K \times L)$; moreover, since the tracks of $H$ are all concatenations of tracks of $\hat{F}$ and $\widehat{G}, A \times B$ stays fixed and tracks of points from $N$ stay in $N$. Letting $J$ be the restriction of $H$ completes the claim.

Corollary 3.3 The product of any two noncompact ARs is inward tame.

Lemma 3.4 Let $X$ and $Y$ be noncompact, simply connected ANRs. Then $X \times Y$ contains arbitrarily small path-connected neighborhoods of infinity, each with a fundamental group that is finitely generated and free. 
Proof Let $U \subseteq X$ and $V \subseteq Y$ be open neighborhoods of infinity, consisting of finitely many unbounded path-connected components $\left\{U_{i}\right\}_{i=1}^{k_{1}}$ and $\left\{V_{j}\right\}_{j=1}^{k_{2}}$, respectively. Then the corresponding rectangular neighborhood of infinity $R=(U \times Y) \cup(X \times V)$ may be covered by the finite collection of path-connected open sets $\left\{U_{i} \times Y\right\}_{i=1}^{k_{1}} \cup$ $\left\{X \times V_{j}\right\}_{j=1}^{k_{2}}$ in which each of the two subcollections is pairwise disjoint, and each $U_{i} \times Y$ intersects each $X \times V_{j}$ in the path-connected set $U_{i} \times V_{j}$. The nerve of this cover is the complete bipartite graph $K_{k_{1}, k_{2}}$ and the connectedness of this graph implies the path connectedness of $R$. A straightforward application of the generalized van Kampen theorem to the corresponding generalized graph of groups [18, Theorem 6.2.11] shows that the fundamental group of $R$ is free on $\left(k_{1}-1\right)\left(k_{2}-1\right)$ generators, the key observation being that each element of a vertex group $\pi_{1}\left(U_{i} \times Y\right)$ can be represented by a loop in $U_{i} \times V_{j}$ which then contracts in $X \times V_{j}$, and similarly for elements of vertex groups $\pi_{1}\left(X \times V_{j}\right)$.

Theorem 3.5 Let $X$ and $Y$ be noncompact, simply connected, movably finitely dominated Hilbert cube manifolds. Then $X \times Y$ is $\mathcal{Z}$-compactifiable.

Proof By a combination of Corollary 3.3, Lemma 3.4 and Theorem 2.3, $X \times Y$ satisfies all conditions of Theorem 2.9.

Theorem 3.6 Let $P_{1}$ and $P_{2}$ be noncompact, simply connected, movably finitely dominated, finite-dimensional, locally finite polyhedra. Then $P_{1} \times P_{2} \times \mathbb{I}^{2\left(\operatorname{dim} P_{1}+\operatorname{dim} P_{2}\right)+5}$ is $\mathcal{Z}$-compactifiable.

Proof Apply Theorems 2.7 and 3.5 to $P_{1} \times P_{2} \times \mathcal{Q}$; then use Theorem 2.10.

\subsection{Spaces admitting homotopically simple $\mathbb{Z}$-actions}

In this section we consider spaces $X$ that admit a proper $\mathbb{Z}$-action generated by a homeomorphism properly homotopic to $\mathrm{id}_{X}$. Under the right circumstances, that hypothesis has significant implication for the topology of $X$.

Lemma 3.7 Let $X$ be an ANR that admits a proper $\mathbb{Z}$-action generated by a homeomorphism $j: X \rightarrow X$ that is properly homotopic to $\mathrm{id}_{X}$. Then:

(1) If the action is cocompact, $X$ is 2 -ended.

(2) If the action is not cocompact, $X$ is 1 -ended.

(3) If $X$ is finitely dominated, then $X$ is inward tame. 
Proof By Lemma 2.6, $(\langle j\rangle \backslash X) \times \mathbb{R} \approx \operatorname{Tor}_{j}(X)$, and since $j \stackrel{p}{\simeq} \mathrm{id}_{X}$, the latter space is proper homotopy equivalent to $X \times \mathbb{S}^{1}$. Now $(\langle j\rangle \backslash X) \times \mathbb{R}$ has either two or one ends, according to whether $\langle j\rangle \backslash X$ is compact or noncompact, and since the number of ends is a proper homotopy invariant, the same is true for $X \times \mathbb{S}^{1}$. Since $X \times \mathbb{S}^{1}$ has the same number of ends as $X$, the first two assertions follow.

Next assume that $X$ is finitely dominated. By Theorem $2.2, X \times \mathbb{S}^{1}$ has finite homotopy type, so by the above equivalences, $\langle j\rangle \backslash X$ also has finite homotopy type. By Lemma 3.1, $(\langle j\rangle \backslash X) \times \mathbb{R}$ is inward tame, and since inward tameness is an invariant of proper homotopy type, $X \times \mathbb{S}^{1}$ is inward tame. It follows that $X$ is inward tame since, if $N$ is a closed neighborhood of infinity in $X$, then $N \times \mathbb{S}^{1}$ is a closed neighborhood of infinity in $X \times \mathbb{S}^{1}$; and a homotopy that pulls $N \times \mathbb{S}^{1}$ into a compact subset projects to a homotopy that pulls $N$ into a compact subset.

Lemma 3.8 Let $X$ be a simply connected ANR that admits a proper $\mathbb{Z}$-action generated by a homeomorphism $j: X \rightarrow X$ that is properly homotopic to $\operatorname{id}_{X}$. Then:

(1) If the action is cocompact, $X$ is simply connected at each of its two ends.

(2) If the action is not cocompact, $X$ is strongly connected at infinity and pro$\pi_{1}(\varepsilon(X))$ is pro-finitely generated free.

Proof The proof is primarily an application of work done in Geohegan and Mihalik [21]; we add a few observations to make those results fit our situation more precisely. For both assertions, we again use the equivalences

$$
(\langle j\rangle \backslash X) \times \mathbb{R} \approx \operatorname{Tor}_{j}(X) \stackrel{p}{\simeq} X \times \mathbb{S}^{1} .
$$

First assume that $\langle j\rangle \backslash X$ is compact. Then $(\langle j\rangle \backslash X) \times \mathbb{R}$ is 2 -ended and the natural choices of base rays, $r_{-}=\{p\} \times(-\infty, 0]$ and $r_{+}=\{p\} \times[0, \infty)$, along with the natural choice of neighborhoods of infinity $(\langle j\rangle \backslash X) \times(-\infty,-n] \cup[n, \infty)$ yield representations of pro- $\pi_{1}\left(\varepsilon((\langle j\rangle \backslash X) \times \mathbb{R}), r_{ \pm}\right)$of the form $\mathbb{Z} \stackrel{\text { id }}{\longleftarrow} \mathbb{Z} \stackrel{\text { id }}{\longleftarrow} \mathbb{Z} \stackrel{\text { id }}{\longleftarrow} \cdots$. The proper homotopy equivalence promised above implies the same for the two ends of $X \times \mathbb{S}^{1}$. Clearly, that can happen only if $X$ is simply connected at each of its two ends.

In the noncocompact case, $(\langle j\rangle \backslash X) \times \mathbb{R}$ is 1 -ended and by [21, Proposition 3.12], with an appropriate choice of base ray, pro- $\pi_{1}(\varepsilon((\langle j\rangle \backslash X) \times \mathbb{R}), r)$ may be represented by an inverse sequence

$$
F_{0} \times\langle a\rangle \stackrel{\lambda_{1} \times \text { id }}{\longleftarrow} F_{1} \times\langle a\rangle \stackrel{\lambda_{2} \times \text { id }}{\longleftarrow} F_{2} \times\langle a\rangle \stackrel{\lambda_{3} \times \text { id }}{\longleftarrow} \cdots,
$$

where each $F_{i}$ is a finitely generated free group, $\lambda_{i}$ takes $F_{i+1}$ onto $F_{i}$ and $\langle a\rangle$ is an infinite cyclic group corresponding to a "copy" of $\pi_{1}\left((\langle j\rangle \backslash X) \times\left\{r_{i}\right\}\right)$, for increasingly 
large $r_{i}$. Semistability of this sequence implies that $(\langle j\rangle \backslash X) \times \mathbb{R}$, and hence $X \times \mathbb{S}^{1}$, is strongly connected at infinity. This allows us to dispense with mention of base rays. It also implies that $X$ is strongly connected at infinity, so pro- $\pi_{1}(\varepsilon(X))$ is semistable and may be represented by an inverse sequence of surjections $H_{0} \stackrel{\mu_{1}}{\longleftarrow} H_{1} \stackrel{\mu_{2}}{\longleftarrow} H_{2} \stackrel{\mu_{3}}{\longleftarrow} \cdots$. It follows that pro- $\pi_{1}\left(\varepsilon\left(X \times \mathbb{S}^{1}\right)\right)$ may be represented by

$$
H_{0} \times\langle t\rangle \stackrel{\mu_{1} \times \text { id }}{\longleftarrow} H_{1} \times\langle t\rangle \stackrel{\mu_{2} \times \text { id }}{\longleftarrow} H_{2} \times\langle t\rangle \stackrel{\mu_{3} \times \text { id }}{\longleftarrow} \cdots,
$$

where each $\langle t\rangle$ is the infinite cyclic group corresponding to the $\mathbb{S}^{1}$-factor.

The equivalences of (3-1) ensure a ladder diagram between subsequences of (3-2) and (3-3). After relabeling to avoid messy subsequence notation, that diagram has the form:

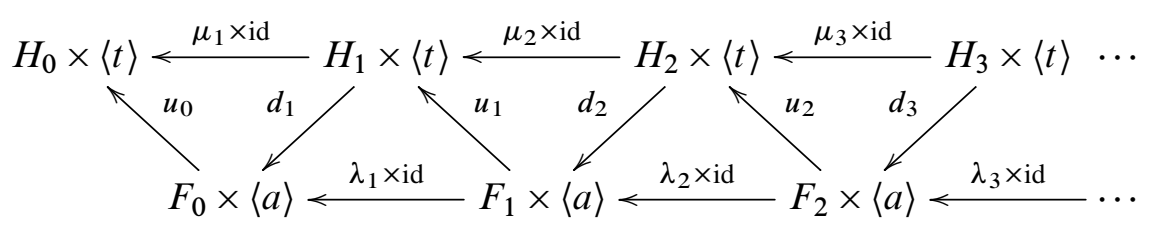

A close look at the homeomorphism between $(\langle j\rangle \backslash X) \times \mathbb{R}$ and $\operatorname{Tor}_{j}(X)$, as described in [19, Section 8], shows that, with appropriate choice of base rays, we may arrange that each $u_{i}$ takes $a$ to $t$. Then, by commutativity, each $d_{i}$ takes $t$ to $a$, each $u_{i}$ takes $F_{i}$ into $H_{i}$ and each $d_{i}$ takes $H_{i}$ into $F_{i-1}$. So diagram (3-4) restricts to a diagram of the form

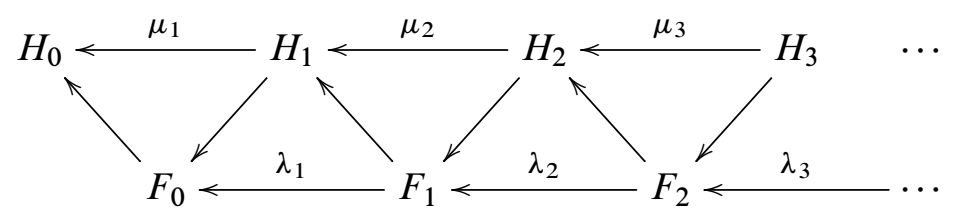

which verifies that pro- $\pi_{1}(\varepsilon(X))$ is pro-finitely generated free.

Theorem 3.9 If a simply connected and finitely dominated Hilbert cube manifold $X$ admits a proper $\mathbb{Z}$-action generated by a homeomorphism $j: X \rightarrow X$ that is properly homotopic to $\operatorname{id}_{X}$, then $X$ is $\mathcal{Z}$-compactifiable.

Proof If the action is not cocompact, then the previous two lemmas together with Theorem 2.3, ensure that $X$ satisfies the conditions of Theorem 2.9. In the cocompact case, the same lemmas imply that $X$ is inward tame and 2-ended, and that each of those ends is simply connected. In order to use the 1-ended version of Theorem 2.9 provided here, split $X$ into a pair of 1-ended Hilbert cube manifolds and apply the theorem to each end individually. 
Theorem 3.10 If a simply connected, locally finite polyhedron $P$ is finitely dominated and finite-dimensional, and admits a proper $\mathbb{Z}$-action generated by a homeomorphism $j: P \rightarrow P$ that is properly homotopic to $\operatorname{id}_{P}$, then $P \times \mathbb{I}^{2 \cdot \operatorname{dim} P+5}$ is $\mathcal{Z}$-compactifiable.

Proof By Theorem 2.7, we have that $j \times \mathrm{id}_{\mathcal{Q}}: P \times \mathcal{Q} \rightarrow P \times \mathcal{Q}$ satisfies the hypotheses of Theorem 3.9. An application of Theorem 2.10 completes the proof.

\subsection{Spaces that are simply connected at infinity}

The key result about Hilbert cube manifolds that are simply connected at infinity is our easiest application of Theorem 2.9; it can be found in Chapman and Siebenmann's original work. For completeness, we include a sketch of their proof.

Theorem 3.11 [6, Corollary to Theorem 8] If $X$ is a Hilbert cube manifold that is simply connected at infinity and $H_{*}(X ; \mathbb{Z})$ is finitely generated, then $X$ is $\mathcal{Z}$ compactifiable.

Sketch of proof Due to the triviality of pro- $\pi_{1}(\varepsilon(X))$, we need only show that $X$ is inward tame. If $N$ is a clean neighborhood of infinity, then $\operatorname{Bd}_{X} N$ is homotopy equivalent to a finite complex. Since $H_{i}\left(\operatorname{Bd}_{X} N ; \mathbb{Z}\right)$ and $H_{i}(X ; \mathbb{Z})$ are both finitely generated for all $i$, and eventually trivial, the Mayer-Vietoris sequence

$$
\cdots \rightarrow H_{i}\left(\operatorname{Bd}_{X} N ; \mathbb{Z}\right) \rightarrow H_{i}(\overline{X-N} ; \mathbb{Z}) \oplus H_{i}(N ; \mathbb{Z}) \rightarrow H_{i}(X ; \mathbb{Z}) \rightarrow \cdots
$$

shows that the same is true for $H_{i}(N ; \mathbb{Z})$. Furthermore, the simple connectivity at infinity of $X$, together with standard techniques from Hilbert cube manifold topology, ensure the existence of arbitrarily small simply connected $N$. Since a simply connected complex with finitely generated $\mathbb{Z}$-homology necessarily has finite homotopy type (see Spanier [33, page 420]), it follows that $X$ is inward tame.

Theorem 3.12 If $P$ is a finite-dimensional, locally finite polyhedron that is simply connected at infinity and $H_{*}(P ; \mathbb{Z})$ is finitely generated, then $P \times \mathbb{I}^{2 \cdot \operatorname{dim} P+5}$ is $\mathcal{Z}$ compactifiable.

Proof Apply Theorems 2.7, 3.11, 2.10. 


\section{Proofs of the main theorems}

We now provide proofs of the unverified theorems from Section 1. Theorems 1.6 and 1.7 require only an assembly of ingredients from Sections 2 and 3, so we begin there.

Proof of Theorem 1.6 Since $\tilde{K}$ is contractible, both $X$ and $Y$ are also contractible. By Lemmas 3.2 and 3.4, $X \times Y$ is inward tame and 1-ended with pro- $\pi_{1}(X \times Y, r)$ that is pro-finitely generated free, and since $\widetilde{K} \stackrel{p}{\simeq} X \times Y$, each of these properties is inherited by $\widetilde{K}$. Applying Theorems $2.7,2.3$ and 2.9 in the usual way provides a $\mathcal{Z}$-compactification of $\widetilde{K} \times \mathcal{Q}$, and since $\operatorname{dim} \widetilde{K}=\operatorname{dim} K<\infty$, Theorem 2.10 provides a $\mathcal{Z}$-compactification of the ER $\tilde{K} \times \mathbb{I}^{2 \cdot \operatorname{dim} K+5}$.

Proof of Theorem 1.7 By Lemmas 3.7 and 3.8, $X$ is inward tame, and either: 2-ended and simply connected at each end; or 1-ended with pro-finitely generated free fundamental group at infinity. By proper homotopy invariance, the same is true for $\tilde{K}$, so by the usual argument, $\widetilde{K} \times \mathcal{Q}$ is $\mathcal{Z}$-compactifiable. Another application of Theorem 2.10 provides a $\mathcal{Z}$-compactification of $\tilde{K} \times \mathbb{I}^{2 \cdot \operatorname{dim} K+5}$.

Remark 5 In the special case, where $X$ (or $\widetilde{K}$ ) admits a cocompact $\mathbb{Z}$-action, the above argument is overkill. There, since $X$ is contractible, $\langle j\rangle \backslash X$ is homotopy equivalent to a circle; and since $\langle j\rangle \backslash X$ compact, a homotopy equivalence $f:\langle j\rangle \backslash X \rightarrow S^{1}$ lifts to a proper homotopy equivalence $X \stackrel{p}{\simeq} \mathbb{R}$. It is then straightforward to show that the 2 -point compactifications of $X$ and $\tilde{K}$ are themselves $\mathcal{Z}$-compactifications.

To obtain the full strength of Theorem 1.8, we require a new ingredient from [19].

Proof of Theorem 1.8 Since $G$ is type $F$, each nontrivial element has infinite order; so we may apply [19, Theorem 1.4] to conclude that $G$ is either simply connected at infinity or $G$ is virtually a surface group. In other words, if $K$ is a finite $\mathrm{K}(G, 1)$ complex, then $\widetilde{K}$ is either simply connected at infinity, or $\widetilde{K}$ is the universal cover of the corresponding finite $\mathrm{K}(H, 1)$ complex $H \backslash \widetilde{K}$, where $H$ is a finite index subgroup of $G$ and $H \cong \pi_{1}(S)$, where $S$ is a closed surface with infinite fundamental group. (Note: by Casson and Jungreis [4] or Gabai [16] a torsion-free virtual surface group is, in fact, a surface group; but that fact is not needed here.)

In the case where $\tilde{K}$ is simply connected at infinity, we may apply Theorem 3.11 to conclude that $\tilde{K} \times \mathcal{Q}$ is $\mathcal{Z}$-compactifiable, and hence $\tilde{K} \times \mathbb{I}^{2 \cdot \operatorname{dim} K+5}$, admits the desired $\mathcal{Z}$-compactification. 
In the second case, we may conclude that $\widetilde{K} \stackrel{p}{\simeq} \widetilde{S} \approx \mathbb{R}^{2}$. It follows that $\widetilde{K}$ is 1 -ended and inward tame, with pro- $\pi_{1}(\varepsilon(\tilde{K}))$ stably isomorphic to $\mathbb{Z}$. By Theorem $2.3, \widetilde{K} \times \mathcal{Q}$ satisfies the hypotheses of Theorem 2.9, and is therefore $\mathcal{Z}$-compactifiable. Another application of Theorem 2.10 completes the proof.

Theorem 1.3 is a special case of Theorem 1.7, so a proof of Theorem 1.2 is all that remains. It is a consequence of Theorem 1.6 and the following crucial lemma.

Lemma 4.1 Let $1 \rightarrow N \rightarrow G \rightarrow Q \rightarrow 1$ be a short exact sequence of groups where both $N$ and $Q$ have type $F$, then $G$ also has type $F$. Moreover, if $Y$ and $Z$ are finite classifying spaces for $N$ and $Q$, respectively, then $G$ admits a finite classifying space $W^{\prime}$ with the property that $\widetilde{W}^{\prime}$ is proper homotopy equivalent to $\tilde{Y} \times \widetilde{Z}$.

The first sentence of this lemma follows from techniques in [18, Sections 6.1, 7.1, 7.2]; the second sentence is essentially a restatement of [18, Proposition 17.3.1]. Due to their importance in this paper, we provide a guide to those arguments in the following outline. Since [18, Proposition 17.3.1] is light on details (and since we had worked out an alternative approach prior to discovering that proposition), we have included an appendix with an alternative proof. A novel aspect of the approach presented there is its use of "approximate fibrations."

Proof of Lemma 4.1 (outline) The construction of a finite $\mathrm{K}(G, 1)$ complex is obtained by an application of the Borel construction followed by the rebuilding lemma; see [18, Section 6.1]. For the Borel construction, begin with a (not necessarily finite) $\mathrm{K}(G, 1)$ complex $X$ and let $G$ act diagonally on $\tilde{X} \times \tilde{Z}$, where the (nonfree) action of $G$ on $\tilde{Z}$ is induced by the quotient map $G \rightarrow Q$. Since the diagonal action itself is free, the quotient $W=G \backslash(\tilde{X} \times \tilde{Z})$ is a $\mathrm{K}(G, 1)$ complex, probably not finite. Inspection of this quotient space reveals a natural projection map $q$ : $W \rightarrow Z$ that is a fiber bundle with fiber the aspherical CW complex $N \backslash \tilde{X}$.

Next is the rebuilding stage of the argument. Here the $\mathrm{K}(G, 1)$ complex $W$ is "rebuilt" by replacing each fiber $N \backslash \tilde{X}$ of the map $q$ : $W \rightarrow Z$ with the homotopy equivalent (but finite) complex $Y$. This is done inductively over the skeleta of $Z$ : first a copy of $Y$ is placed over each vertex of $Z$, then over each edge $e$ of $Z$ a copy of $Y \times[0,1]$ is attached with $Y \times 0$ being glued to the copy of $Y$ lying over the initial vertex of $e$ and $Y \times 1$ glued to the copy of $Y$ lying over the terminal vertex of $e$. From there we move to the 2-cells of $Z$, and so on. At each step, the bundle map $q$ provides instructions for the gluing maps. At the end we have a bundle-like "stack" of CW complexes $q^{\prime}: W^{\prime} \rightarrow Z$ with each point preimage a copy of $Y$ and a homotopy 
equivalence $k: W^{\prime} \rightarrow W$. Since both $Z$ and $Y$ are finite complexes, $W^{\prime}$ is a finite complex, so $G$ has type F.

Obtaining a proper homotopy equivalence $h: \widetilde{W}^{\prime} \rightarrow \tilde{Y} \times \widetilde{Z}$ is an interesting and delicate task. A proof can be found in the appendix; otherwise, the reader is referred to Geoghegan [18, Proposition 17.3.1].

\section{Appendix: An alternative approach to Lemma 4.1}

In this appendix we take a closer look at the proper homotopy equivalence promised in Lemma 4.1 and offer an alternative to the proof suggested in [18]. Begin with a short exact sequence of groups $1 \rightarrow N \rightarrow G \rightarrow Q \rightarrow 1$ where both $N$ and $Q$ have type F. Then, as described in the sketched proof of Lemma 4.1, $G$ also has type F. More specifically, if $Y$ is a finite $\mathrm{K}(N, 1)$ complex and $Z$ is a finite $\mathrm{K}(Q, 1)$ complex, then there is a finite $\mathrm{K}(G, 1)$ complex $W^{\prime}$, obtained by an application of the Borel construction followed by the rebuilding lemma. As a corollary of the construction, $W^{\prime}$ comes equipped with a map $q: W^{\prime} \rightarrow Z$ for which each point preimage is a copy of $Y$. In fact, for each open $k$-cell $\stackrel{\circ}{k}^{k}$ of $Z, q^{-1}\left(e^{k}\right) \approx \stackrel{\circ}{e}^{k} \times Y$.

Although $q$ : $W^{\prime} \rightarrow Z$ is not necessarily a fiber bundle, it exhibits many properties of a fiber bundle; it is a stack of CW complexes over $Z$ with fiber $Y$. If we let $\widehat{W}^{\prime}$ be the intermediate cover of $W^{\prime}$ corresponding to $N \unlhd G$, we get another stack of CW complexes $\widehat{q}: \widehat{W}^{\prime} \rightarrow \widetilde{Z}$ over the contractible space $\widetilde{Z}$. Given the standard fact that a fiber bundle over a contractible space is always a product bundle, it is reasonable to hope that, in the case at hand, $\widehat{W}^{\prime}$ is "approximately a product." By using the aptly named theory of "approximate fibrations," we will eventually arrive at the following main result of this appendix.

Proposition A.1 Given the above setup, $\widehat{W}^{\prime}$ is proper homotopy equivalent to $Y \times \tilde{Z}$.

This result is stronger than needed to complete Lemma 4.1, and may be of interest in its own right. Lemma 4.1 is obtained from Proposition A.1 by lifting the promised proper homotopy equivalence to the universal covers. It is worth noting that $W^{\prime}$, itself, is typically not homotopy equivalent to $Y \times Z$.

In this appendix, we first provide a constructive proof of the special case where $Q$ is infinite cyclic; in that case $G$ is a semidirect product $G \cong N \rtimes_{\varphi} \mathbb{Z}$. The special case motivates the work to be done later and also illustrates the subtleties that are overcome with the general theory. After completing the special case, we will provide a brief overview of the theory of "approximate fibrations." Then we employ that theory to prove Proposition A.1 in full generality. 


\section{A.1 Mapping tori of self-homotopy equivalences}

In this section we focus on the special case of Proposition A.1, where $G$ is an extension of the form $1 \rightarrow N \rightarrow G \rightarrow \mathbb{Z} \rightarrow 1$; equivalently, $G \cong N \rtimes_{\varphi} \mathbb{Z}$ for some automorphism $\varphi: G \rightarrow G$. In this case, the Borel/rebuilding procedure yields a finite $\mathrm{K}(G, 1)$ complex that is the mapping torus of a map $f: Y \rightarrow Y$, with $f_{\#}=\varphi$. Since $Y$ is a $\mathrm{K}(H, 1), f$ is necessarily a homotopy equivalence. The goal of this section then becomes the following.

Lemma A.2 If $K$ is a compact connected ANR and $f: K \rightarrow K$ is homotopy equivalence, then the canonical infinite cyclic cover, $\operatorname{Tel}_{f}(K)$, of $\operatorname{Tor}_{f}(K)$ is proper homotopy equivalent to $K \times \mathbb{R}$.

Proof Let $g: K \rightarrow K$ be a homotopy inverse for $f$ and $B: K \times[0,1] \rightarrow K$ with $B_{0}=\mathrm{id}_{K}$ and $B_{1}=f g$. In accordance with Lemma 2.4, our goal is to define a map $u: K \times \mathbb{R} \rightarrow \operatorname{Tel}_{f}(K)$, such that there is a proper strong deformation retraction of $\mathcal{M}_{[0,1]}(u)$ onto the domain copy of $K \times \mathbb{R}$. For each integer $n$, define a function $u_{n}: K \times[n, n+1] \rightarrow \mathcal{M}_{[n, n+1]}(f)$ by the rules

$$
\begin{cases}u_{n}(x, r)=q_{[n, n+1]}\left(B_{r-n}\left(g^{n}(x)\right), r\right) & \text { when } n \geq 0, \\ \left.u_{n}(x, r)=q_{[n, n+1]}\left(f^{-n}(x), r\right)\right) & \text { when } n<0 .\end{cases}
$$

Here it is understood that $g^{0}=\mathrm{id}_{K}$.

Note that

$$
\left.u_{-1}(x, 0)=q_{[-1,0]}(f(x), 0)\right) \quad \text { while } \quad u_{0}(x, 0)=q_{[0,1]}\left(B_{0}(x), 0\right)=q_{[0,1]}(x, 0),
$$

and for each integer $n \geq 1$,

$$
\begin{aligned}
u_{n-1}(x, n) & =q_{[n-1, n]}\left(B_{1}\left(g^{n-1}(x)\right)\right)=q_{[n-1, n]}\left(f g g^{n-1}(x), n\right)=q_{[n-1, n]}\left(f g^{n}(x), n\right), \\
u_{n}(x, n) & =q_{[n, n+1]}\left(B_{0}\left(g^{n}(x)\right), n\right)=q_{[n, n+1]}\left(g^{n}(x), n\right) .
\end{aligned}
$$

Similarly, for each for each integer $n \leq-1$,

$$
\begin{aligned}
u_{n-1}(x, n) & \left.=q_{[n-1, n]}\left(f^{-(n-1)}(x), n\right)\right)=q_{[n-1, n]}\left(f\left(f^{-n}(x), n\right)\right), \\
u_{n}(x, n) & \left.=q_{[n, n+1]}\left(f^{-n}(x), n\right)\right) .
\end{aligned}
$$

It follows that the $u_{n}$ can be glued together to obtain a proper map $u: K \times \mathbb{R} \rightarrow \operatorname{Tel}_{f}(K)$. See Figure 1.

Claim There is a proper strong deformation retraction of $\mathcal{M}_{[0,1]}(u)$ onto $K \times \mathbb{R}$. 


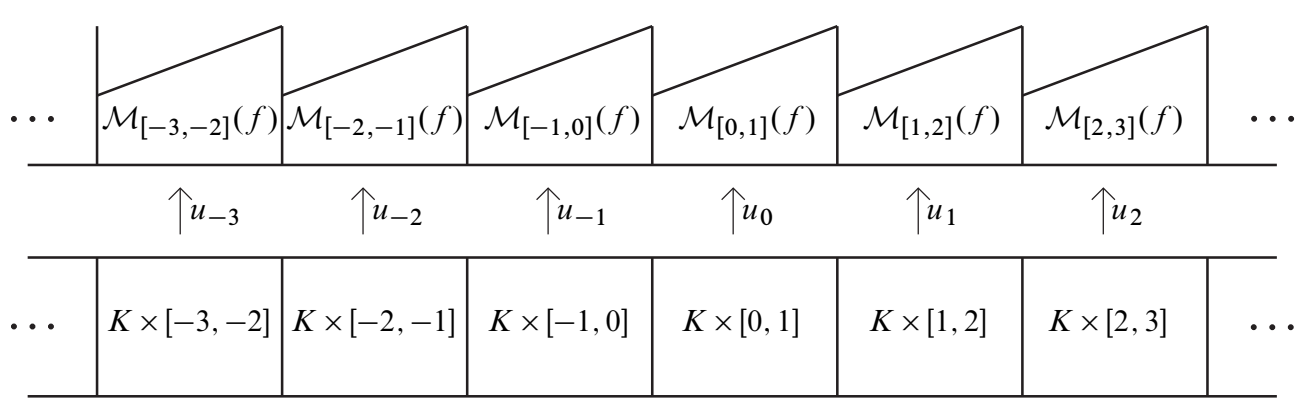

Figure 1: The map $u: K \times \mathbb{R} \rightarrow \operatorname{Tel}_{f}(K)$

First note that, since $u$ respects $\mathbb{R}$-coordinates, the natural projections $K \times \mathbb{R} \rightarrow \mathbb{R}$ and $p: \operatorname{Tel}_{f}(K) \rightarrow \mathbb{R}$ can be extended to a projection $\hat{p}: \mathcal{M}_{[0,1]}(u) \rightarrow \mathbb{R}$ with the property that each point preimage $\hat{p}^{-1}(r)$ is a mapping cylinder $C_{r}$ of a map from $K \times\{r\}$ to $K_{r}$. Indeed, for an integer $n \geq 0, C_{n}$ is the mapping cylinder of $f g^{n}$ and for an integer $n<0, C_{n}$ is the mapping cylinder of $f^{-(n-1)}$. So each $C_{n}$ is a mapping cylinder of a homotopy equivalence; a fact that will be useful later. (In fact, each $C_{r}$ is a mapping cylinder of a homotopy equivalence, but this fact will only be used for integral values of $r$.) Note also that $\mathcal{M}_{[0,1]}(u)$ may be viewed as a countable union $\bigcup_{n \in \mathbb{Z}} \mathcal{M}_{[0,1]}\left(u_{n}\right)$, where each $\mathcal{M}_{[0,1]}\left(u_{n}\right)$ intersects $\mathcal{M}_{[0,1]}\left(u_{n-1}\right)$ in $C_{n}$.

Subclaim For each $n, \mathcal{M}_{[0,1]}\left(u_{n}\right)$ strong deformation retracts onto the subset $C_{n} \cup$ $(K \times[n, n+1])_{1} \cup C_{n+1}$.

It suffices to show that $C_{n} \cup(K \times[n, n+1])_{1} \cup C_{n+1} \hookrightarrow \mathcal{M}\left(u_{n}\right)$ is a homotopy equivalence. Since $C_{n}$ and $C_{n+1}$ are mapping cylinders of homotopy equivalences, each strong deformation retracts onto its domain end, so $(K \times[n, n+1])_{1} \hookrightarrow C_{n} \cup$ $(K \times[n, n+1])_{1} \cup C_{n+1}$ is a homotopy equivalence; therefore, it is enough to show that $(K \times[n, n+1])_{1} \hookrightarrow \mathcal{M}_{[0,1]}\left(u_{n}\right)$ is a homotopy equivalence. Note that the inclusions $K_{n} \hookrightarrow C_{n}, K_{n} \hookrightarrow \mathcal{M}_{[n, n+1]}(f)$ and $\mathcal{M}_{[n, n+1]}(f) \hookrightarrow \mathcal{M}_{[0,1]}\left(u_{n}\right)$ are all homotopy equivalences, since each subspace is the range end of a corresponding mapping cylinder. It follows that $C_{n} \hookrightarrow \mathcal{M}_{[0,1]}\left(u_{n}\right)$ is a homotopy equivalence, and since $K \times\{n\} \hookrightarrow C_{n}$ is a homotopy equivalence it follows that $K \times\{n\} \hookrightarrow \mathcal{M}_{[0,1]}\left(u_{n}\right)$, and hence $(K \times[n, n+1])_{1} \hookrightarrow \mathcal{M}_{[0,1]}\left(u_{n}\right)$ is a homotopy equivalence. The subclaim follows.

To complete the claim, properly strong deformation retract $\mathcal{M}_{[0,1]}(u)$ onto $(K \times \mathbb{R})_{1} \cup$ $\left(\cup_{n \in \mathbb{Z}} C_{n}\right)$ using the union of the strong deformation retractions provided by the subclaim. Follow that by a strong deformation of $(K \times \mathbb{R})_{1} \cup\left(\cup_{n \in \mathbb{Z}} C_{n}\right)$ onto $(K \times \mathbb{R})_{1}$ obtained by individually strong deformation retracting each $C_{n}$ onto its domain end. 
Remark 6 The delicate nature of defining $u: K \times \mathbb{R} \rightarrow \operatorname{Tel}_{f}(K)$ in the above proof hints at the subtlety of Lemma 4.1 .

\section{A.2 Approximate fibrations}

We now review the main definitions and a few fundamental facts from the theory of approximate fibrations: a theory developed by Coram and Duvall $[9 ; 10]$ to generalize the notions of fibration and fiber bundle.

A proper surjective map $p: E \rightarrow B$ between (locally compact, metric) ANRs is an approximate fibration if it satisfies the following approximate lifting property:

For every homotopy $H: X \times[0,1] \rightarrow B$, map $h: X \rightarrow E$ with $\pi h=H_{0}$ and open cover $\mathcal{U}$ of $B$, there exists $\bar{H}: X \times[0,1] \rightarrow E$ such that $\bar{H}_{0}=h$ and $p \bar{H}$ is $U$-close to $H$ (that is, for each $(x, t) \in X \times[0,1]$ there exists $U \in \mathcal{U}$ containing both $H(x, t)$ and $p \bar{H}(x, t))$.

For each $b \in B, F_{b}:=p^{-1}(b)$ is called a fiber. Approximate fibrations allow for fibers with bad local properties; however, the theory is easier, but still rich, when fibers are ANRs. Since fibers of the maps considered in this paper are always ANRs (in fact, finite CW complexes), we will focus on that special case. In this context, there is a particularly nice criterion for recognizing an approximate fibration.

Suppose $p: E \rightarrow B$ is a proper map between connected ANRs with ANR fibers. Then, for each fiber $F_{b}$, some neighborhood $U_{b}$ retracts onto $F_{b}$, and for points $b^{\prime}$ sufficiently close to $b$, this induces a map of $F_{b^{\prime}}$ to $F_{b}$. By [10], $p: E \rightarrow B$ is an approximate fibration if and only if each $b \in B$ has a neighborhood over which each of these induced fiber maps is a homotopy equivalence.

Example 4 If $f: K \rightarrow K$ is homotopy equivalence of a compact connected ANR to itself, the above criterion is easily applied to show that quotient maps $p_{1}: \mathcal{M}_{[a, b]}(f) \rightarrow$ $[a, b], p_{2}: \operatorname{Tor}_{f}(K) \rightarrow \mathbb{S}^{1}$ and $p_{3}: \operatorname{Tel}_{f}(K) \rightarrow \mathbb{R}$ are all approximate fibrations. In the simple case where $K$ is an arc and $f$ is a constant map, these projections are not actual fibrations. In the case where $K$ is a bouquet of circles and $f$ is an arbitrary map inducing a $\pi_{1}$-isomorphism, the examples are already of group-theoretic interest.

As with the case of ordinary fibrations, approximate fibrations give rise to homotopy long exact sequences [9]. When no restrictions are placed on the fibers, these sequence involve the shape (or Čech) homotopy groups of the fiber; when the fibers are ANRs that technicality vanishes and we have the following lemma. 
Lemma A.3 Let $p: E \rightarrow B$ be an approximate fibration between connected ANRs with connected ANR fibers. Then, for any $b \in B$, there is a long exact sequence

$$
\cdots \longrightarrow \pi_{k+1}(B) \longrightarrow \pi_{k}\left(F_{b}\right) \stackrel{i_{\#}}{\longrightarrow} \pi_{k}(E) \stackrel{p_{\#}}{\longrightarrow} \pi_{k}(B) \longrightarrow \pi_{k-1}\left(F_{b}\right) \longrightarrow \cdots,
$$

where $i$ is the inclusion map.

We now prove a general fact about approximations that is almost tailor-made for proving Proposition A.1.

Theorem A.4 Let $p: E \rightarrow B$ be an approximate fibration between connected ANRs with connected ANR fibers and let $b \in B$. If $B$ is contractible then $E$ is proper homotopy equivalent to $F_{b} \times B$.

Proof By the homotopy long exact sequence and an application of the Whitehead Theorem, $F_{b} \stackrel{i}{\hookrightarrow} E$ is a homotopy equivalence. Let $r_{b}: E \rightarrow F_{b}$ be a homotopy inverse that retracts $E$ onto $F_{b}$. We will observe that $r_{b} \times p: E \rightarrow F_{b} \times B$ is a proper homotopy equivalence.

Clearly $r_{b} \times p$ is proper, and by contractibility of $B$, it is a homotopy equivalence. We will show that it is a proper homotopy equivalence by exhibiting cofinal sequences of neighborhoods of infinity in the domain and range, respectively, such that $r_{b} \times p$ restricts to homotopy equivalences between corresponding entries. An application of the proper Whitehead Theorem [18, Theorem 17.1.1] or Siebenmann [32, Proposition IV] (applied to the mapping cylinder of $r_{b} \times p$ ) completes the proof.

Let $\left\{V_{i}\right\}_{i=0}^{\infty}$ be a cofinal nested sequence of neighborhoods of infinity in $B$. For convenience, assume that $B$ is 1 -ended and that each $V_{i}$ is chosen to be connected; we will return to the general case momentarily. For each $i$, let $U_{i}=p^{-1}\left(V_{i}\right)$. Then $\left\{U_{i}\right\}$ and $\left\{F_{b} \times V_{i}\right\}$ are nested cofinal sequence of neighborhoods of infinity in $E$ and $F_{b} \times B$, respectively. Moreover, $r_{b} \times p$ restricts to a map of $U_{i}$ to $F_{b} \times V_{i}$, for each $i$.

Note that the restriction $p_{i}: U_{i} \rightarrow V_{i}$ is, itself, an approximate fibration. By choosing $b$ to lie in $V_{i}$, and recalling that the composition $F_{b} \hookrightarrow U_{i} \hookrightarrow E$ induces $\pi_{k}-$ isomorphisms, for all $k$, we see that the long exact sequence for $p_{i}: U_{i} \rightarrow V_{i}$ yields a short exact sequence

$$
1 \longrightarrow \pi_{k}\left(F_{b}\right) \stackrel{i_{\#}}{\longrightarrow} \pi_{k}\left(U_{i}\right) \stackrel{p_{i \#}}{\longrightarrow} \pi_{k}\left(V_{i}\right) \longrightarrow 1
$$

for each $k$. Since $U_{i}$ retracts onto $F_{b}$, these sequences split; so we have $\pi_{k}\left(U_{i}\right) \cong$ $\pi_{k}\left(F_{b}\right) \times \pi_{k}\left(V_{i}\right)$. From there it is easy to see that each restriction of $r_{b} \times p$ induces isomorphisms $\pi_{k}\left(U_{i}\right) \rightarrow \pi_{k}\left(F_{b} \times V_{i}\right)$, completing the proof. 
If $B$ has more than one end, one simply applies the above argument to individual components of the $V_{i}$.

Proof of Proposition A.1 By Theorem A.4, it suffices to show that the stack of CW complexes $\widehat{q}: \widehat{W}^{\prime} \rightarrow \widetilde{Z}$ is an approximate fibration. Since each point preimage is a copy of the finite complex $Y$, we need only check that these fibers "line up homotopically" in the sense of the approximate fibration recognition criterion described above.

From the initial Borel/rebuilding construction, it is clear that the inclusion of each fiber into $\widehat{W}^{\prime}$ indices a $\pi_{1}$-isomorphism; and, since both $Y$ and $\widehat{W}^{\prime}$ are aspherical, these inclusions are homotopy equivalences. So any retraction of $\widehat{W}^{\prime}$ onto a fiber $Y$ restricts to homotopy equivalences between fibers. The recognition criterion follows.

\section{References}

[1] H Bass, A Heller, R G Swan, The Whitehead group of a polynomial extension, Inst. Hautes Études Sci. Publ. Math. (1964) 61-79 MR0174605

[2] M Bestvina, Local homology properties of boundaries of groups, Michigan Math. J. 43 (1996) 123-139 MR1381603

[3] M Bestvina, G Mess, The boundary of negatively curved groups, J. Amer. Math. Soc. 4 (1991) 469-481 MR1096169

[4] A Casson, D Jungreis, Convergence groups and Seifert fibered 3-manifolds, Invent. Math. 118 (1994) 441-456 MR1296353

[5] T A Chapman, Lectures on Hilbert cube manifolds, Regional Conf. Ser. Math. 28, Amer. Math. Soc. (1976) MR0423357

[6] T A Chapman, L C Siebenmann, Finding a boundary for a Hilbert cube manifold, Acta Math. 137 (1976) 171-208 MR0425973

[7] M M Cohen, A course in simple-homotopy theory, Graduate Texts Mat. 10, Springer, New York (1973) MR0362320

[8] G Conner, M Mihalik, Commensurated subgroups, semistability and simple connectivity at infinity arXiv:1201.2965

[9] D S Coram, P F Duvall, Jr, Approximate fibrations, Rocky Mountain J. Math. 7 (1977) 275-288 MR0442921

[10] D Coram, P Duvall, Approximate fibrations and a movability condition for maps, Pacific J. Math. 72 (1977) 41-56 MR0467745

[11] A N Dranishnikov, On Bestvina-Mess formula, from: "Topological and asymptotic aspects of group theory", (R Grigorchuk, M Mihalik, M Sapir, Z Šuniḱ, editors), Contemp. Math. 394, Amer. Math. Soc. (2006) 77-85 MR2216707 
[12] J Dugundji, Topology, Allyn and Bacon, Boston, MA (1966) MR0193606

[13] R D Edwards, Characterizing infinite-dimensional manifolds topologically (after Henryk Toruńczyk), from: "Séminaire Bourbaki 1978/79 (Exposé 540)", Lecture Notes in Math. 770, Springer, Berlin (1980) 278-302 MR572429

[14] F T Farrell, J-F Lafont, EZ-structures and topological applications, Comment. Math. Helv. 80 (2005) 103-121 MR2130569

[15] S C Ferry, Stable compactifications of polyhedra, Michigan Math. J. 47 (2000) 287-294 MR1793625

[16] D Gabai, Convergence groups are Fuchsian groups, Ann. of Math. 136 (1992) 447-510 MR1189862

[17] R Geoghegan, The shape of a group_connections between shape theory and the homology of groups, from: "Geometric and algebraic topology", (H Toruńczyk, S Jackowski, S Spiez, editors), Banach Center Publ. 18, PWN, Warsaw (1986) 271-280 MR925870

[18] R Geoghegan, Topological methods in group theory, Graduate Texts in Mathematics 243, Springer, New York (2008) MR2365352

[19] R Geoghegan, C R Guilbault, Topological properties of spaces admitting free group actions, J. Topol. 5 (2012) 249-275 MR2928076

[20] R Geoghegan, M L Mihalik, Free abelian cohomology of groups and ends of universal covers, J. Pure Appl. Algebra 36 (1985) 123-137 MR787167

[21] R Geoghegan, M L Mihalik, The fundamental group at infinity, Topology 35 (1996) 655-669 MR1396771

[22] D H Gottlieb, A certain subgroup of the fundamental group, Amer. J. Math. 87 (1965) 840-856 MR0189027

[23] C R Guilbault, Ends, shapes, and boundaries in manifold topology and geometric group theory arXiv:1210.6741

[24] C R Guilbault, A non-Z-compactifiable polyhedron whose product with the Hilbert cube is $\mathcal{Z}$-compactifiable, Fund. Math. 168 (2001) 165-197 MR1852740

[25] C R Guilbault, Products of open manifolds with $\mathbb{R}$, Fund. Math. 197 (2007) 197-214 MR2365887

[26] C R Guilbault, F C Tinsley, Manifolds with non-stable fundamental groups at infinity, II, Geom. Topol. 7 (2003) 255-286 MR1988286

[27] O Hanner, Some theorems on absolute neighborhood retracts, Ark. Mat. 1 (1951) 389-408 MR0043459

[28] B Jackson, End invariants of group extensions, Topology 21 (1982) 71-81 MR630881

[29] M Mather, Counting homotopy types of manifolds, Topology 3 (1965) 93-94 MR0176470 
[30] M L Mihalik, Solvable groups that are simply connected at $\infty$, Math. Z. 195 (1987) 79-87 MR888128

[31] J S Profio, Using subnormality to show the simple connectivity at infinity of a finitely presented group, Trans. Amer. Math. Soc. 320 (1990) 281-292 MR961627

[32] L C Siebenmann, Infinite simple homotopy types, Indag. Math 32 (1970) 479-495 MR0287542

[33] E H Spanier, Algebraic topology, Springer, New York (1981) MR666554 Corrected reprint

[34] C T C Wall, Finiteness conditions for CW-complexes, Ann. of Math. 81 (1965) 56-69 MR0171284

[35] J E West, Mapping Hilbert cube manifolds to ANR's: A solution of a conjecture of Borsuk, Ann. of Math. 106 (1977) 1-18 MR0451247

Department of Mathematical Sciences, University of Wisconsin-Milwaukee PO Box 413, Milwaukee, WI 53201, USA

craigg@uwm.edu

Received: 23 August 2013 